\title{
Activation of antigen-presenting cells by microbial products breaks self tolerance and induces autoimmune disease
}

\author{
Hanspeter Waldner, ${ }^{1}$ Mary Collins, ${ }^{2}$ and Vijay K. Kuchroo ${ }^{3}$
}

${ }^{1}$ Center for Neurologic Diseases, Brigham and Women's Hospital and Harvard Medical School, Cambridge, Massachusetts, USA. ${ }^{2}$ Wyeth Research, Cambridge, Massachusetts, USA. ${ }^{3}$ Center for Neurologic Diseases, Brigham and Women's Hospital and Harvard Medical School, Boston, Massachusetts, USA

\begin{abstract}
We describe the generation of mice that express a transgenic $T$ cell receptor (TCR) (5B6) specific for the encephalitogenic myelin proteolipid protein (PLP) peptide 139-151, on the experimental autoimmune encephalomyelitis-resistant (EAE-resistant) B10.S background. Despite harboring a high frequency of selfreactive $T$ cells, $5 \mathrm{~B} 6$ transgenic mice on the $\mathrm{B} 10 . S$ background rarely develop spontaneous $E A E$, which is in striking contrast to $5 \mathrm{~B} 6$ transgenic mice on the EAE-susceptible SJL background. The relative resistance to spontaneous EAE in transgenic B10.S mice is not due to deletion or anergy of T cells, but appears to be controlled by APCs. Analysis of APCs revealed a lower activation state and a lower T cell-activating capacity for APCs from B10.S mice than for those from EAE-susceptible SJL mice. When APCs in 5B6 transgenic B10.S mice were activated, for example, via TLR9 or TLR4, $T$ cell tolerance was broken, resulting in EAE. Our findings demonstrate that activation of APCs via innate immune receptors can break self tolerance and trigger the development of autoimmunity even in a genetically resistant strain. These findings suggest that the development of autoimmune diseases such as multiple sclerosis is determined at least partly by the endogenous activation state of APCs.
\end{abstract}

\section{Introduction}

It has become clear that the genetic composition of a host partly determines the specificity and intensity of autoimmune disease. Genetic factors including MHC and non-MHC genes have been shown to be involved in determining susceptibility or tolerance to autoimmunity in experimental autoimmune encephalomyelitis (EAE) and type 1 diabetes in NOD mice, the mouse models of multiple sclerosis and juvenile diabetes, respectively (1-4). Tolerance to autoimmune disease can be achieved by a variety of reported mechanisms. Negative selection of self-reactive $T$ cells in the thymus deletes most but not all $\mathrm{T}$ cells with specificity against self antigens, thereby reducing the size and affinity of the autoreactive $\mathrm{T}$ cell repertoire (5). Some autoreactive $T$ cells escape thymic deletion and can be found in the periphery, where they are normally kept under check by mechanisms of peripheral tolerance. The mechanisms responsible for peripheral $\mathrm{T}$ cell tolerance include "ignorance" of self antigens, anergy or unresponsiveness to autoantigen, cytokine immune deviation, and activation-induced cell death of self-reactive $\mathrm{T}$ cells (6). In addition, regulatory $\mathrm{T}$ cells have been shown to be involved in mediating peripheral tolerance (7).

B10.S mice share the same MHC with SJL mice but are relatively resistant to the induction of EAE by immunization with different myelin antigens, including myelin basic protein (MBP) and proteolipid protein (PLP) peptide 139-151 (8-9). The relative resistance of B10.S mice to MBP-induced EAE has been associated at least part-

Nonstandard abbreviations used: bone marrow (BM); experimental autoimmune encephalomyelitis (EAE); incomplete Freund's adjuvant (IFA); myelin basic protein (MBP); oligodeoxynucleotide (ODN); pertussis toxin (PTX); phycoerythrin (PE); proteolipid protein (PLP); recombinant murine ( $\mathrm{rm})$; specific pathogen-free $(\mathrm{SPF})$; T cell receptor (TCR)

Conflict of interest: The authors have declared that no conflict of interest exists.

Citation for this article: J. Clin. Invest. 113:990-997 (2004).

doi:10.1172/JCI200419388. ly with a lack of development of encephalitogenic Th1 cells and lack of production of proinflammatory cytokines (IFN- $\gamma$ ) by T cells (10).

We have recently generated transgenic mice that express the rearranged $\mathrm{T}$ cell receptor (TCR) genes from a PLP139-151-specific $\mathrm{CD} 4^{+} \mathrm{T}$ cell clone (5B6), which develop fulminant spontaneous EAE on the EAE-susceptible SJL background (11). To examine mechanisms that may be responsible for self tolerance in autoimmune resistant mice, we have crossed the PLP TCR transgenic mice onto the B10.S background. This approach allowed us to study the development of PLP139-151-specific T cells in autoimmune-resistant B10.S mice, in contrast to previous studies in nontransgenic mice in which specific $T$ cells could be generated only after antigen immunizations.

Whereas 5B6 TCR transgenic SJL mice readily develop EAE spontaneously, in this report we show that 5B6 TCR transgenic mice on the EAE-resistant B10.S strain rarely develop spontaneous EAE. The transgenic $T$ cells from these mice are not deleted in the thymus or the periphery and are fully functional when activated by antigen in vitro. However, in comparison with cells from the EAE-susceptible SJL strain, antigen-presenting cells (APCs) from B10.S mice show lower expression of MHC class II molecules and stimulate $5 \mathrm{~B} 6$ transgenic $\mathrm{T}$ cells less efficiently in vitro. Furthermore, activation of APCs via the innate immune receptor TLR9 or TLR4 breaks self tolerance and results in the induction of EAE in 5B6 transgenic mice on the EAE-resistant B10.S background. These data suggest a critical role for APCs in maintaining self tolerance and in genetic susceptibility to EAE.

\section{Methods}

Mice. 5B6 transgenic mice specific for myelin PLP139-151 were generated and characterized as previously described (11). The transgenic founder line 1858 was backcrossed for at least five generations onto B10.S mice (McLaughlin Research Inst., Great Falls, Montana, USA). 
Recombination-activating gene 2-deficient (RAG-2-/-) B10.S mice were generated by backcrossing of the RAG-2 deficiency onto the B10.S strain for ten generations and were maintained by intercrosses in our facility. 5B6 transgenic $R A G-2^{-/-}$mice were obtained by crossing of $5 \mathrm{~B} 6$ transgenic B10.S mice with $R A G-2^{-/-}$B10.S mice and intercrosses of transgenic litters. Mice were housed at the Harvard Institutes of Medicine specific pathogen-free/viral antibody-free (SPF/VAF) animal facility in accordance with the guidelines of Harvard Medical School. Female mice were used at 6-12 weeks of age except for the induction of EAE with LPS, for which equal numbers of female and male mice were used.

Flow cytometry. Single-cell suspensions of spleens and thymi were prepared by straining of the tissues through cell strainers. Red blood cells were lysed by hypotonic shock. Thymocytes or spleen cells $\left(1 \times 10^{6} /\right.$ sample $)$ were washed in $50 \mu$ flow cytometry buffer (PBS, $0.1 \% \mathrm{NaN}_{3}$, and 1\% FCS) and stained with FITC-conjugated or phycoerythrin-conjugated (PE-conjugated) antibodies for 20-30 minutes at $4^{\circ} \mathrm{C}$. Cells were subsequently washed twice in flow cytometry buffer before they were analyzed on a FACS model flow cytometer (BD Biosciences, San Jose, California, USA). At least 30,000 live cells per sample were analyzed. Dead cells were excluded by gating on characteristic forward-and side-scatter profiles. Antibodies conjugated to the indicated fluorochromes were purchased from PharMingen (San Diego, California, USA) unless noted otherwise and included anti-CD4 (RM4-5), anti-CD8 (536.7), anti-TCR V 66 (RR4-7), anti-CD11b (M1/70), anti-CD11c (HL3), anti-CD19 (1D3), anti-CD86 (GL1), anti-F4/80 (A3-1; Serotec, Raleigh, North Carolina, USA), and biotinylated anti I-Aq (KH116; cross-reacts with I-As).

Peptide antigens. The following peptide antigens were used: PLP139-151 (HSLGKWLGHPDKF) and PLP178-191 (NTWTTCQSIAFPSK). The peptides were synthesized by Quality Controlled Biochemicals (Hopkinton, Massachusetts, USA) and were more than $90 \%$ pure as determined by HPLC.

Oligodeoxymucleotides (ODNs). The following ODNs were purchased from Operon Technologies (Almeda, California, USA): CpG ODN 1760 (5'-ATAATCGACGTTCAAGCAAG-3') and non-CpG ODN 1908 (5'-ATAATAGAGCTTCAAGCAAG-3') (CpG motifs and inverted non-CPG motifs are underlined) (12). ODNs were phosphorothioated throughout their sequences to increase their resistance to nuclease degradation.

Stimulation of T cells. Spleen cell samples from 5B6 transgenic mice were enriched for $\mathrm{CD}^{+} \mathrm{T}$ cells by negative selection using CD4 $\mathrm{T}$ cell enrichment columns (Cytovax, Edmonton, Alberta, Canada) according to the manufacturer's protocol. Spleen cells $\left(4 \times 10^{5} /\right.$ well $)$ or CD4-enriched $\mathrm{T}$ cell samples $\left(1 \times 10^{5}\right.$ cells/well $)$ together with irradiated $(5,000 \mathrm{rad})$ splenocytes $\left(5 \times 10^{5} /\right.$ well $)$ from nontransgenic littermates were cultured in triplicates in 96-well plates in RPMI 1640 medium (BioWhittaker, Walkersville, Maryland, USA) supplemented with $10 \%$ heat-inactivated FCS, 2 mM L-glutamine, 10 $\mathrm{mM}$ HEPES, and $2 \mathrm{mM}$ mercaptoethanaol (RPMI complete) in the presence of different concentrations of synthetic PLP139-151 or control peptide PLP178-191 and were incubated for 4 days at $37^{\circ} \mathrm{C}$. Proliferation of responding cells was determined by incorporated thymidine after the addition of $1 \mu \mathrm{Ci}$ of $\left[{ }^{3} \mathrm{H}\right]$ thymidine to each well during the last 12 hours of culture.

DAS cells (I-As-transfected fibroblasts, expressing B7.1; provided by S. Miller, Northwestern University, Evanston, Illinois, USA) were pulsed with $10 \mu \mathrm{g} / \mathrm{ml} \mathrm{PLP} 139-151$ for 2 hours at $37^{\circ} \mathrm{C}$ and were used as APCs to stimulate CD4-enriched T cell samples $\left(4 \times 10^{5}\right.$ cells/well) from 5B6 transgenic mice for 72 hours. Proliferation of T cells was subsequently determined as described above.

For examination of the efficiency with which APCs stimulate $\mathrm{T}$ cells, T cell samples from $5 \mathrm{~B} 6$ transgenic SJL mice enriched for $\mathrm{CD}^{+}$ cells (CD3 columns; R\&D Systems, Minneapolis, Minnesota, USA) $\left(2 \times 10^{5}\right.$ cells/well $)$ were cultured with titrated numbers of irradiated (3,300 rad) spleen cells from nontransgenic SJL or B10.S mice $(0$ $\times 10^{5}$ to $5 \times 10^{5}$ cells/well $)$ in the presence of PLP139-151 $(10 \mu \mathrm{g} / \mathrm{ml})$ for 72 hours. T cell proliferation was assessed as described above.

Stimulation of APCs with CPG ODN. For stimulation of APCs with CpG ODN, spleen cells $\left(4 \times 10^{5} /\right.$ well $)$ from nontransgenic B10.S mice were cultured in triplicate with $1.5 \mu \mathrm{M} C \mathrm{PG}$ or non-CpG ODN for 48 hours, washed and stained with antibodies against indicated surface molecules.

ELISA. The concentration of cytokines was determined in culture supernatants of spleen cells or CD4-enriched T cell samples in presence of irradiated splenocytes from nontransgenic littermate mice following antigen stimulation. Supernatants of these cultures were assayed for cytokine production after 40 hours by quantitative capture ELISA according to the manufacturers' guidelines. Assays were developed with TMB Microwell Peroxidase Substrate (Kirkegaard and Perry Laboratories, Gaithersburg, Maryland, USA) and were "read" at $450 \mathrm{~nm}$.

In vitro generation of APCs. Bone marrow-derived (BM-derived) APCs were prepared according to the protocol of Lutz et al. (13). Briefly, BM cells from B10.S or SJL mice were cultured for 10-13 days in RPMI complete medium supplemented with $20 \mathrm{ng} / \mathrm{ml}$ recombinant murine GM-CSF (rmGM-CSF; PeproTech, Rocky Hill, New Jersey, USA). The medium was replaced every 3 days, and at the end of culture, adherent cells were detached using trypsin-EDTA (Invitrogen, Carlsbad, California, USA). The cells were subsequently washed in PBS and analyzed by flow cytometry.

Immunizations and assessment of EAE. 5B6 TCR transgenic B10.S mice were injected subcutaneously with CPG ODN 1760 or nonCpG ODN 1908 (10 nmol each) in the presence of PLP139-151 (25 $\mu \mathrm{g})$ emulsified in an equal volume of incomplete Freund's adjuvant (IFA) (Sigma-Aldrich) or with CpG ODN 1760 (10 nmol) in IFA alone. Mice that were immunized with $\mathrm{CPG}$ ODN only were readministered CPG ODN 1760 (10 nmol) in PBS by intraperitoneal injection 29 days later. LPS (from Escherichia coli 055:B5; $50 \mu \mathrm{g}$ ) or rmIL-12 (300 ng; Genetics Institute/Wyeth, Cambridge, Massachusetts, USA) in PBS was injected intraperitoneally into mice once or every other day in 11 administrations. Pertussis toxin (PTX) (75 ng; List Biological Laboratories, Campbell, California, USA) was administered intravenously on days 0 and 2 . Mice were monitored and assigned grades for clinical signs of EAE for at least 44 days (70 days for CPG ODN group) using the following scoring system: 0 , healthy; 1 , limp tail; 2 , impaired righting reflex or waddled gait; 3 , hind limb paralysis; 4, total limb paralysis; 5, moribund.

\section{Results}

Low incidence of spontaneous EAE in 5 B6 transgenic B10.S mice. The 5B6 TCR was derived from an encephalitogenic $\mathrm{CD}^{+} \mathrm{T}$ cell clone generated from lymph node cells of an SJL mouse immunized with PLP139-151 (14). Mice transgenic for the 5B6 TCR were generated on the FVB background, backcrossed onto the EAE-susceptible SJL and EAE-resistant B10.S backgrounds and were monitored routinely for the development of spontaneous EAE. The incidence of spontaneous EAE was strikingly different in the two strains studied. While a mean of $40 \% 5 \mathrm{~B} 6$ transgenic mice on the SJL strain 


\section{Table 1}

Low incidence of spontaneous EAE in 5B6 TCR transgenic B10.S mice

$\begin{array}{lcc}\text { Generation } & \text { B10.S } & \text { SJL } \\ \text { N4 } & 0 / 9(0 \%) & 6 / 9(67 \%) \\ \text { N5 } & 0 / 2(0 \%) & 8 / 28(29 \%) \\ \text { N5 F1 } & 0 / 13(0 \%) & \text { N.D. } \\ \text { N6 } & 3 / 42(7 \%) & 9 / 20(45 \%) \\ \text { N6 F1 } & 0 / 7(0 \%) & \text { N.D. } \\ \text { Mean } & 3 / 73(4 \%) & 23 / 57(40 \%)\end{array}$

5B6 transgenic B10.S and SJL backcross generations were monitored regularly for clinical signs of EAE for at least 6 months. Numbers of transgenic mice with spontaneous disease per total number of 5B6 transgenic mice in each generation are shown, and resulting incidences of spontaneous EAE are given (\%). F1 designates the offspring of the intercross between transgenic mice of the same backcross generation. N.D., not determined.

developed spontaneous EAE, only 4\% transgenic mice on the B10.S background developed disease when monitored over the same backcross generations and time period (Table 1). The 5B6 transgenic B10.S mice that did develop spontaneous EAE showed mild signs of clinical disease with a mean disease severity of 1.3 (data not shown). The significantly lower incidence of spontaneous EAE in transgenic B10.S mice could not be explained by exposure to different environmental antigen, as the SJL and B10.S colonies were housed under identical conditions in the same room of our animal facility, which is maintained under sterile SPF conditions. Taken together, these results show that in contrast to $5 \mathrm{~B} 6$ transgenic SJL mice, B10.S transgenic mice were relatively resistant to development of spontaneous EAE, as overall only a small percentage of transgenic mice developed mild clinical signs of EAE spontaneously.

$T$ cell development in $5 B 6$ transgenic B10.S mice. To determine whether the relative resistance to spontaneous EAE observed in the $5 \mathrm{~B} 6$ transgenic B10.S mice could be attributed to the deletion of autoreactive $T$ cells either in the thymus or periphery, we examined the $T$ cell populations in these mice by flow cytometry. Thymocytes from 5B6 transgenic B10.S mice were skewed toward the singlepositive $\mathrm{CD} 4$ population at the expense of $\mathrm{CD}^{+} \mathrm{T}$ cells (Figure 1A). A pronounced skewing toward the $\mathrm{CD}^{+} \mathrm{T}$ cell population was expected, as the transgenic TCR genes were isolated from a MHC class II-restricted $\mathrm{CD}^{+} \mathrm{T}$ cell clone. Consistent with earlier results with TCR transgenic systems (15), this pattern is a strong indication that the transgenic T cells were positively selected. Essentially more than $95 \% \mathrm{CD}^{+}$splenic T cells expressed the transgene-encoded TCR V $\beta 6$ chain in 5B6 transgenic B10.S mice (Figure 1B). The transgenic TCR V $\beta 6$ chain on CD $4^{+}$splenocytes was expressed at a physiological level, as the expression level of endogenous V $\beta 6$ in nontransgenic littermates was very similar (Figure 1B). The total number of peripheral $\mathrm{T}$ cells in $5 \mathrm{~B} 6$ transgenic mice was similar to that of nontransgenic controls (approximately $38 \times 10^{6} \mathrm{vs} .34 \times 10^{6}$ cells), indicating that there was no gross deletion of self-reactive $T$ cells in the $5 \mathrm{~B} 6$ transgenic B10.S mice.

Allelic exclusion of the TCR $\alpha$ chain is less tightly controlled than that of the TCR $\beta$ chain, resulting in the expression of nontransgenic TCR $\alpha$ chains paired with transgenic TCR $\beta$ chains. We could not directly examine the extent of endogenous TCR V $\alpha$ expression in the $5 \mathrm{~B} 6$ transgenic B10.S mice due to the lack of a specific antibody. However, we assessed the percentage of $\mathrm{CD}^{+} \mathrm{T}$ cells in peripheral blood expressing nontransgenic TCR V $\alpha$ chains by staining with available antibodies against TCR V $\alpha 2, \operatorname{V\alpha } 3.2, \mathrm{~V} \alpha$, and $V \alpha 11$. Assuming that there was no preferential pairing of 5B6 transgenic TCR $\beta$ chains with a nontransgenic TCR $\alpha$ chains, we estimated that $90 \%$ of peripheral $\mathrm{CD}^{+} \mathrm{T}$ cells in B10.S mice bear the transgenic TCR complex (data not shown). To further substantiate the finding that the majority of $\mathrm{CD} 4^{+} \mathrm{T}$ cells expressed the transgenic TCR and to conclusively determine whether the transgenic $T$ cells were efficiently selected on the autoimmuneresistant B10.S background, we examined $\mathrm{T}$ cell populations in thymi and spleens of 5B6 transgenic B10.S mice that were deficient in RAG-2. In thymi of $5 \mathrm{~B} 6$ transgenic $R A G-2^{-/-}$mice, which expressed the transgenic TCR exclusively, $\mathrm{T}$ cells were not deleted but were efficiently selected to the $\mathrm{CD}^{+}{ }^{+}$compartment (Figure $1 \mathrm{~A}$ ). The efficient positive selection by the transgenic TCR seen in the thymus was reflected in the periphery, where $80 \%$ of splenocytes were $\mathrm{CD}^{+}$and expressed the transgenic 5B6 TCR. The absolute numbers of $\mathrm{CD} 4^{+} \mathrm{V} \beta 6^{+} \mathrm{T}$ cells in $R A G-2^{-/-}$transgenic mice and normal transgenic mice were comparable, confirming the above estimations that expression of endogenous TCRs in the transgenic B10.S mice was minor (Figure 1B).

In summary, the transgenic 5B6 TCR-expressing T cells were not deleted but were efficiently selected on the autoimmune-resistant B10.S background. Thus, neither central nor peripheral deletion of transgenic T cells was the mechanism responsible for resistance to spontaneous EAE in these mice.

$T$ cells from $5 B 6$ transgenic B10.S mice are not anergic but respond to PLP139-151. To determine whether T cells from 5B6 transgenic B10.S mice responded as efficiently as T cells isolated from 5B6 transgenic SJL mice, we stimulated T cells purified from spleen cells of the transgenic mice with PLP139-151 presented by DAS cells (I-A $\mathrm{A}^{\mathrm{s}}$-transfected fibroblasts) as artificial APCs. T cells from both 5B6 transgenic SJL and B10.S mice showed a strong and comparable proliferative response to PLP139-151 (Figure 2A). These data demonstrate that T cells from 5B6 transgenic SJL and B10.S
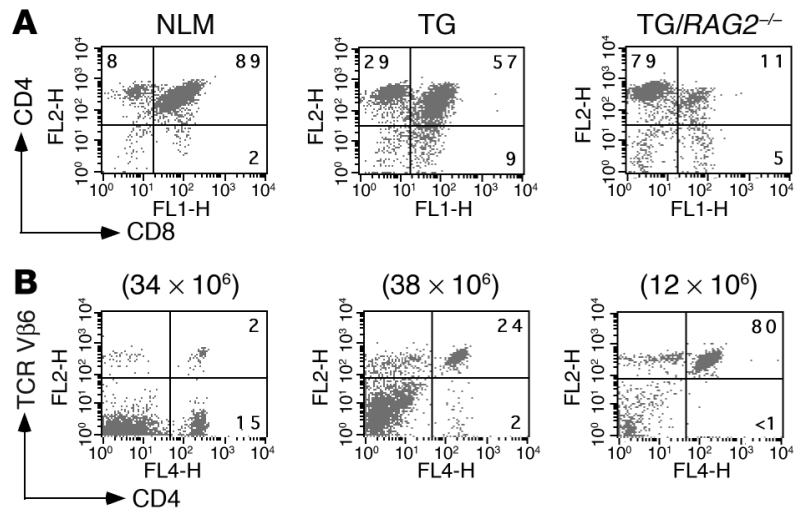

\section{Figure 1}

Flow cytometry analysis of thymocytes and peripheral T cells in 5B6 transgenic B10.S mice. (A and B) T cells from thymi (A) and spleens (B) of mice were stained with the indicated antibodies (PE- or allophycocyanin-conjugated anti-CD4, FITC-conjugated anti-CD8, and PEconjugated anti-TCR V $\beta 6$ ). Dot plots representing two-color flow cytometry analysis of 5B6 transgenic mice with or without RAG-2 deficiency (TG/RAG-2 ${ }^{-/-}$or TG, respectively) and nontransgenic littermates (NLM) are shown. Numbers in quadrants refer to percentages of gated cell populations. Total numbers of spleen cells are shown above dot plots. 
mice have a comparable capacity to proliferate in response to PLP139-151 presented by neutral APCs.

We next asked whether T cells from 5B6 transgenic B10.S mice were functional when stimulated by antigen presented by syngeneic APCs. Thus, we determined the proliferative and cytokine responses of $\mathrm{CD}^{+} \mathrm{T}$ cells from 5B6 transgenic B10.S mice after stimulation by PLP139-151 in vitro. Splenocyte samples enriched for $\mathrm{CD}^{+} \mathrm{T}$ cells from unimmunized $5 \mathrm{~B} 6$ transgenic B10.S mice proliferated vigorously when stimulated with PLP139-151 presented by syngeneic nontransgenic splenocytes as APCs (Figure 2B). This response was highly antigen specific, since the $T$ cells failed to proliferate to the control I-A ${ }^{\mathrm{s}}$ binding peptide PLP178-191. In contrast, PLP139-151-specific proliferation was not detected in CD4 ${ }^{+} \mathrm{T}$ cells isolated from naive nontransgenic littermates (Figure 2B). In comparison with B10.S T cells, CD4-enriched T cell samples from 5B6 transgenic SJL mice demonstrated a similar proliferative response following antigen stimulation (Figure 2B). To determine whether immune deviation to an anti-inflammatory cytokine profile could explain the relative tolerance to spontaneous EAE in the 5B6 transgenic B10.S mice, we examined the cytokine response of transgenic $\mathrm{T}$ cells following stimulation with PLP139-151 in vitro. Supernatants of PLP139-151-stimulated CD4+-enriched T cell cultures were assayed for secretion of IL-2, IL-4, IL-10, TNF- $\alpha$, and IFN- $\gamma$ by ELISA. We consistently found that $5 \mathrm{~B} 6$ transgenic $\mathrm{CD}^{+} \mathrm{T}$ cells stimulated with PLP139-151 produced IL-2 in significant amounts, in contrast to $\mathrm{T}$ cells from nontransgenic littermates (Figure $2 \mathrm{C}$ and data not shown). The amounts of IL-2 detected in the culture supernatants of $\mathrm{CD}^{+} \mathrm{T}$ cells from $5 \mathrm{~B} 6$ transgenic SJL mice were generally lower than those in cultures from transgenic B10.S mice. The amounts of IL-4, IL-10, INF- $\gamma$, and TNF- $\alpha$ were generally below the detection limit in supernatants from both the transgenic and negative littermate cultures, indicating that $\mathrm{T}$ cells from $5 \mathrm{~B} 6$ transgenic B10.S mice had a naive phenotype. Because we did not detect INF- $\gamma$ in culture supernatants of purified CD4 T cells from 5B6 transgenic B10.S and SJL mice, we examined the production of this cytokine by whole spleen cells after stimulation with PLP139-151. Significant and comparable amounts of INF- $\gamma$ production in PLP139-151-stimulated spleen cell cultures of 5B6 B10.S or SJL mice were observed (Figure 2D). This observation indicates that spleen cells from 5B6 transgenic B10.S mice can mount antigen-specific INF- $\gamma$ responses. Since purified transgenic $\mathrm{CD}^{+} \mathrm{T}$ cells did not produce INF- $\gamma$ upon antigen-specific stimulation, the production of INF- $\gamma$ in spleen cell cultures suggests that this cytokine is produced by an alternative source such as APCs, CD8 T cells, or NK cells. Taken together, these data demonstrate that $\mathrm{T}$ cells from $5 \mathrm{~B} 6$ transgenic B10.S mice were not anergic but were highly responsive to PLP139-151 presented by either artificial or syngeneic APCs and showed antigen-specific responses comparable to those of $\mathrm{T}$ cells from 5B6 transgenic SJL mice.

APCs in B10.S mice show a lower basal state of activation and stimulate PLP-specific T cells less efficiently than do SJL APCs. Based on our findings above, we concluded that the relative resistance to spontaneous EAE in the 5B6 transgenic B10.S mice could not be explained as being due to thymic deletion or peripheral tolerance of transgenic T cells. To determine whether the failure of 5B6 transgenic T cells in B10.S mice to mediate spontaneous EAE was due to a defect in APCs, we examined the activation/maturation state and responsiveness of APCs in wild-type B10.S mice.

One of the hallmarks of activation and maturation of APCs such as dendritic cells (DCs) is upregulation of MHC class II and cos- timulatory molecules (16). Thus, we examined the activation/maturation state of APCs in wild-type B10.S mice by determining the expression level of I-As on subsets of APCs, as defined by CD19+ (B cells), CD11b ${ }^{+}, \mathrm{CD} 11 \mathrm{c}^{+}$(DCs), and F4/80 (macrophages). The activation/maturation state of APCs from B10.S mice was compared with that of APCs from EAE-susceptible SJL mice. As expected in unimmunized mice, the examined subsets of APCs expressed low to moderate surface levels of MHC class II. Within each APC subset, the number of MHC class $\mathrm{II}^{+}$cells was lower in B10.S than in SJL wild-type mice (Figure 3A). Furthermore, APCs from B10.S mice consistently expressed lower levels of MHC class II than did those from SJL mice, as determined by the mean fluorescence intensity (Figure 3, B and C). We also examined the activation state of BM-derived APCs by culturing BM from wild-type SJL and B10.S mice with GM-CSF and assessing surface expression of MHC class II on $\mathrm{CD}_{11 \mathrm{~b}^{+}}, \mathrm{CD} 11 \mathrm{c}^{+}$, or $\mathrm{F} 4 / 80^{+}$cells. The generation of APCs in vitro yielded lower numbers of MHC class II-expressing APC subsets from B10.S BM than from SJL BM (Figure 3D). In agreement
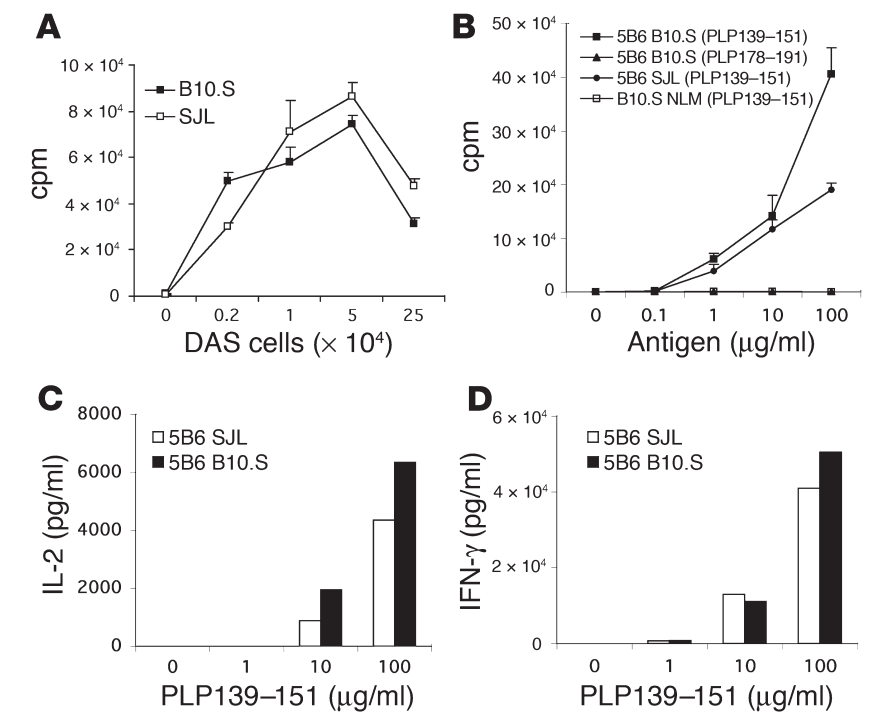

\section{Figure 2}

Responses of 5B6 transgenic T cells to PLP139-151. (A) Proliferative response of 5B6 transgenic T cells to PLP139-151 presented by DAS cells. CD4-enriched T cell samples from 5B6 transgenic SJL and B10.S mice were stimulated with the indicated numbers of PLP139-151-pulsed DAS cells. T cell proliferation was assessed by $\left[{ }^{3} \mathrm{H}\right]$ thymidine incorporation assay. The mean $\mathrm{cpm} \pm \mathrm{SD}$ of triplicate cultures are shown. (B) Proliferative response of T cells from $5 \mathrm{~B} 6$ transgenic B10.S or SJL mice to PLP139-151 presented by syngeneic APCs. Splenocyte samples from unimmunized 5B6 transgenic and nontransgenic littermate (NLM) B10.S or SJL mice were enriched for CD4+ $\mathrm{T}$ cells and were cultured with irradiated splenocytes from nontransgenic littermates in the presence of the indicated concentrations of PLP139-151 or control peptide PLP178-191. Proliferative responses were determined by $\left[{ }^{3} \mathrm{H}\right]$ thymidine incorporation assay. The mean $\mathrm{cpm} \pm \mathrm{SD}$ of triplicate cultures of one experiment representative of three are shown. (C) IL-2 response to PLP139-151 of T cells from 5B6 transgenic B10.S or SJL mice. Supernatants from cultures in $\mathbf{B}$ were assayed in duplicate by ELISA for cytokine production. Representative data from one of three experiments are shown. (D) INF- $\gamma$ response of spleen cells from $5 B 6$ transgenic B10.S or SJL mice to PLP139-151 stimulation. Culture supernatants from 5B6 transgenic SJL or B10.S whole spleen cells stimulated with the indicated concentrations of PLP139-151 were assayed in duplicate by ELISA for INF- $\gamma$ production. 
A
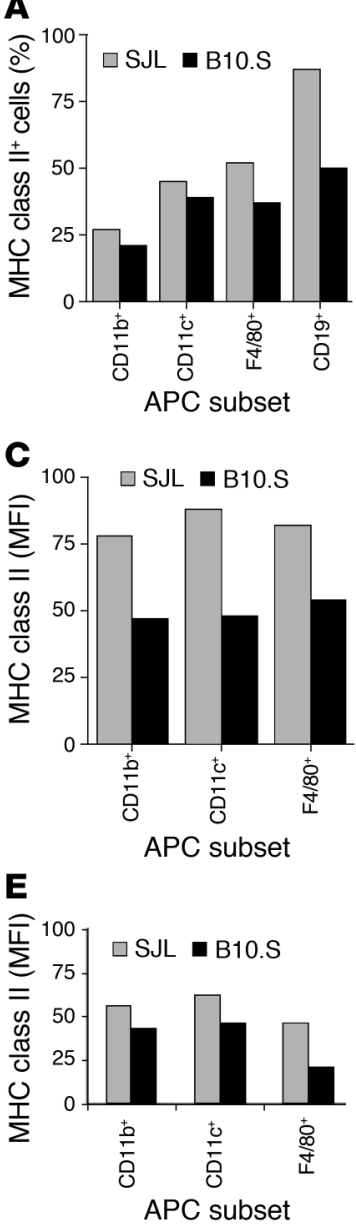

B

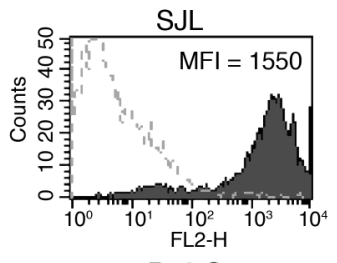

B10.S
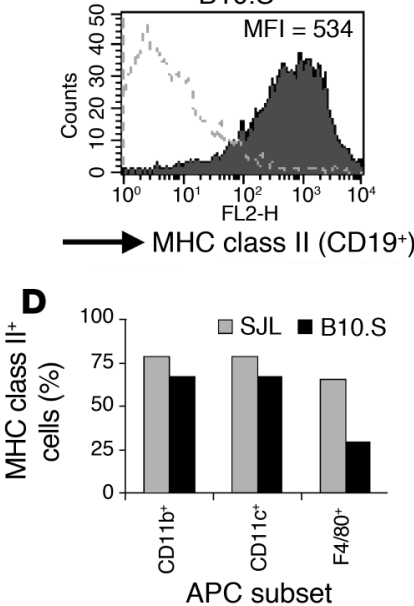

$\mathbf{F}$

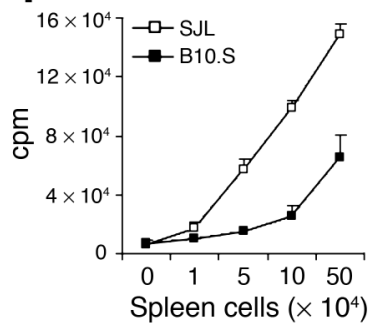

with the data from ex vivo APCs, the activation/maturation state, as assessed by MHC class II expression, of BM-derived CD11 $\mathrm{b}^{+}$, $\mathrm{CD} 11 \mathrm{c}^{+}$and $\mathrm{F} 4 / 80^{+} \mathrm{APCs}$ from B10.S mice was lower than that of APCs from SJL mice (Figure 3E).

To determine the functional significance of the low activation/maturation state of APCs from B10.S mice compared with SJL APCs, we examined their ability to stimulate $5 \mathrm{~B} 6$ transgenic $\mathrm{T}$ cells in proliferation assays. Purified T cells from $5 \mathrm{~B} 6$ transgenic SJL mice were stimulated with irradiated spleen cells as APCs from nontransgenic SJL or B10.S mice in presence of PLP139-151. APCs from B10.S mice were less efficient in stimulating 5B6 transgenic $\mathrm{T}$ cells to proliferate than were APCs from SJL mice at constant concentrations of PLP139-151 (Figure 3F). This difference in $\mathrm{T}$ cell-activating capacity between the APCs from the two strains was seen at different $\mathrm{APC} / \mathrm{T}$ cell ratios, indicating that it was APC dependent. However, it was not dependent on the source of the transgenic $\mathrm{T}$ cells, as similar results were obtained in proliferation assays using T cells from $5 \mathrm{~B} 6$ transgenic B10.S mice (data not shown). These data indicate that antigen-pulsed APCs from B10.S mice are less efficient in inducing $\mathrm{T}$ cell proliferation than are APCs from SJL mice. In summary, splenic APCs from wild-type B10.S mice show lower activation/maturation states and induce a lower proliferative response in $5 \mathrm{~B} 6$ transgenic $\mathrm{T}$ cells than do wild-type SJL APCs.

$A P C s$ from B10.S mice are activated by $C p G O D N s$. It has recently been shown that synthetic ODNs that contain unmethylated CPG

\section{Figure 3}

Activation/maturation state and T cell-activating capacity of APCs. (A-E) Spleen cells (A-C) or BM-derived APCs ( $\mathbf{D}$ and $\mathbf{E})$ from wild-type B10.S and SJL mice were stained with PE-conjugated antibodies against the indicated subsets of APCs and biotinylated anti-I-A $q / \mathrm{s}$. The level of MHC class II expression was determined on gated APC subsets as indicated (\%) (A and $\mathbf{D})$ by flow cytometry and is shown as mean of fluorescence intensity (MFI) (B, C, and E). Data from one of three experiments with similar data are shown. $(\mathbf{F})$ The indicated numbers of irradiated spleen cells from wild-type SJL or B10.S mice as APCs in presence of PLP139-151 were incubated with 5B6 transgenic SJL T cells and were subsequently pulsed with $\left[{ }^{3} \mathrm{H}\right]$ thymidine. T cell proliferation was measured by $\left[{ }^{3} \mathrm{H}\right]$ thymidine incorporation assay. Values are shown as mean $\mathrm{cpm} \pm \mathrm{SD}$ of triplicate wells. One experiment representative of three is shown.

motifs (CpG ODNs) trigger activation and maturation of murine APCs such as dendritic cells, macrophages, and B cells through the Toll-like receptor TLR9 (17-19). To exclude the possibility that APCs from B10.S mice have a general defect in activation, we determined whether they could be activated in vitro by sequence-specific CPG ODNs. CPG ODN induced upregulation of MHC class II and $\mathrm{CD} 86$ on $\mathrm{CD} 19^{+}, \mathrm{CD} 11 \mathrm{~b}^{+}$, and $\mathrm{CD} 11 \mathrm{c}^{+}$subsets of spleen cells from B10.S mice and expanded the examined APC subsets compared with stimulation with non-CpG ODN (Figure 4). Taken together, these data demonstrate that $\mathrm{CPG}$ ODNs can expand and activate B10.S APC populations in vitro, as determined by upregulation of MHC class II and the costimulatory molecule CD86.

In vivo activation of APCs induces EAE in $5 B 6$ transgenic mice on the resistant B10.S background. To examine the role of APCs in EAE, we next asked whether $\mathrm{CPG}$ ODN-mediated activation of APCs could break self tolerance and induce $\mathrm{EAE}$ in the $5 \mathrm{~B} 6$ transgenic B10.S mice. Administration of CPG ODN alone, which activates APCs through TLR9 (19), was sufficient to break T cell tolerance and induce EAE in 33\% of 5B6 transgenic B10.S mice (Table 2). However, administration of $\mathrm{CPG}$ ODN in presence of low amounts of PLP139-151/IFA induced severe EAE in the majority of $5 \mathrm{~B} 6$ transgenic B10.S mice (75\%). In striking contrast, when
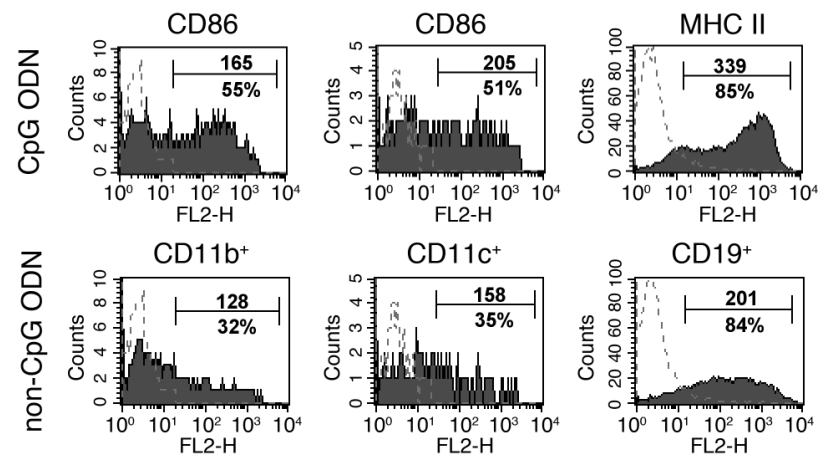

\section{Figure 4}

CpG ODN induces expansion of APC subsets and upregulation of MHC class II and CD86. Spleen cells from B10.S mice were stimulated with CpG ODN or non-CpG ODN. Spleen cells were subsequently stained with FITC-conjugated anti-CD11b, anti-CD11c, and anti-CD19 and PEconjugated anti-CD86 or biotinylated anti-I-A q/s. Expression of CD86 and $\mathrm{MHC}$ class II was determined on CD11C $\mathrm{C}^{+}$, CD11 b+-, or CD19+gated cells. Dotted lines indicate isotype controls. Numbers refer to mean of fluorescence intensity of specific staining (upper number) shown on gated APC subsets (\%). 
Table 2

Immunization of 5B6 transgenic B10.S mice with CpG ODN induces EAE

$\begin{array}{lccc}\text { Immunization } & \text { Incidence } & \begin{array}{c}\text { Mean day } \\ \text { of onset }\end{array} & \begin{array}{c}\text { Mean peak } \\ \text { severity }\end{array} \\ \text { CpG } & 3 / 10(33 \%) & 43 \pm 22 & 3.3 \pm 1.5 \\ \text { CpG + PLP139-151 } & 9 / 12(75 \%) & 35 \pm 13 & 3.0 \pm 1.2 \\ \text { Non-CpG + PLP139-151 } & 0 / 8(0 \%) & \mathrm{N} / \mathrm{A} & \mathrm{N} / \mathrm{A}\end{array}$

5B6 transgenic B10.S mice were injected with $10 \mathrm{nmol}$ each of $\mathrm{CpG}$ ODN in IFA alone; CPG ODN with $25 \mu \mathrm{g}$ PLP139-151 in IFA; or nonCpG ODN with $25 \mu \mathrm{g}$ PLP139-151 in IFA. Mice were monitored for clinical signs of EAE for at least 50 days ( 70 days for CpG/IFA group). Data were pooled from three independent experiments.

non-CpG ODN plus PLP139-151 was administered, that is, in absence of TLR9-mediated APC activation, none of the 5B6 transgenic mice developed any clinical signs of EAE. These findings indicate that even in presence of exogenous autoantigen, activation of APCs is critical for development of autoimmune disease in this TCR transgenic model.

To determine whether abrogation of self tolerance in 5B6 transgenic B10.S mice was specific to the activation of APCs via TLR9, we examined whether systemic administration of microbial agents such as LPS and PTX also resulted in EAE in these mice. LPS activates DCs to release IL-12 by signaling through TLR4 and induces INF- $\gamma$-secreting CD $4^{+} \mathrm{T}$ cells in vivo $(20,21)$. $5 \mathrm{~B} 6$ transgenic B10.S mice injected with LPS showed a low incidence rate of $13 \%$, resulting in an earlier onset of very mild EAE (Table 3). None of the nontransgenic littermate control mice developed any signs of clinical EAE (data not shown). PTX, an inhibitor of $G \alpha_{i}$ proteins with diverse physiological effects such as breaching the blood-brain barrier and promoting lymphocytosis $(22,23)$, has recently been shown to induce INF- $\gamma$ responses in T cells in absence of IL-12 via activation of APCs $(24,25)$. Administration of PTX to 5 B 6 transgenic B10.S mice resulted in EAE (mean peak severity, $2.6 \pm 0.3$ ) in all of the treated animals with clinical signs starting at 6 days (Table 3 ). The PTX-induced EAE was specific for the 5B6 TCR transgenic mice, as none of the nontransgenic littermate controls showed any signs of clinical EAE. IL-12 is thought to play a crucial role in induction of INF- $\gamma$ production by $\mathrm{T}$ cells (26). To directly elucidate the role of IL-12 in breaking $\mathrm{T}$ cell tolerance in our transgenic model, we treated 5B6 transgenic B10.S mice every other day with rmIL-12 in PBS for a total of 11 administrations. Over the monitoring period of 44 days, only one IL-12-treated mouse out of six developed clinical EAE, with delayed onset compared with PTX- or LPS-induced EAE. In contrast, none of the 5B6 transgenic B10.S mice that were injected with PBS as controls developed any signs of clinical EAE (data not shown).

Taken together, our results indicate that in vivo activation of APCs via the innate immune receptor TLR9 could break self tolerance and induce EAE in the 5B6 transgenic B10.S mice. Activation of APCs via TLR4 or administration of IL-12 to induce INF- $\gamma$ production by $\mathrm{T}$ cells was less effective in inducing EAE in these mice than was TLR9-mediated APC activation. In contrast, PTX, which has pleiotropic effects on the immune system inducing APC activation, was more effective in mediating EAE in 5B6 transgenic B10.S mice than was CPG ODN.

\section{Discussion}

In this study we have examined mechanisms of tolerance to the development of spontaneous EAE in EAE-resistant B10.S mice that are transgenic for a PLP139-151-specific TCR. We have shown that despite large numbers of myelin-specific $T$ cells, they fail to mediate spontaneous EAE in the majority of 5B6 transgenic B10.S mice in contrast to $5 \mathrm{~B} 6$ transgenic mice on the EAE-susceptible SJL background. Several mechanisms of $\mathrm{T}$ cell tolerance in the thymus or periphery that could account for the low incidence of spontaneous EAE in transgenic B10.S mice were considered. Failure of positive selection or clonal deletion of transgenic $T$ cells was not apparent, as mature $\mathrm{CD} 4^{+} \mathrm{T}$ cells were efficiently selected and at least $90 \%$ of these cells expressed the transgene-specific 5B6 TCR $\alpha / \beta$ chain in the periphery (Figure 1 ). CD4-enriched T cell samples from transgenic B10.S mice were not anergic, as they proliferated and secreted IL-2 in a dose-dependent response to PLP139-151 and comparably to $\mathrm{CD}^{+} \mathrm{T}$ cells from $5 \mathrm{~B} 6$ transgenic SJL mice. Although $\mathrm{CD}^{+}{ }^{+} \mathrm{T}$ cells from $5 \mathrm{~B} 6$ transgenic B10.S and SJL mice did not produce detectable amounts of INF- $\gamma$, whole spleen cell cultures showed substantial and comparable amounts of INF- $\gamma$ following antigen stimulation. These findings indicate that splenic APCs are essential for induction of INF- $\gamma$ production by $T$ cells or, alternatively, that the INF- $\gamma$ detected in whole spleen cell cultures is not $\mathrm{T}$ cell derived but is produced by other cell types such as APCs, CD8 ${ }^{+} \mathrm{T}$ cells, or NK cells. When antigen was presented by artificial APCs (DAS cells) in vitro, T cells from both 5B6 transgenic SJL and B10.S mice showed a comparable antigen-specific proliferative response (Figure 2). Thus, on both the susceptible SJL background and the resistant B10.S background, the T cells are fully capable of responding to PLP139-151 in a comparable fashion.

Transfer of $\mathrm{CD}^{+} \mathrm{CD} 25^{+}$-depleted spleen cell samples into nude mice has been shown to induce a variety of autoimmune diseases, indicating a regulatory role of $\mathrm{CD} 4^{+} \mathrm{CD} 25^{+} \mathrm{T}$ cells in preventing autoimmunity. The number of $\mathrm{CD} 4^{+} \mathrm{CD} 25^{+}$regulatory $\mathrm{T}$ cells is a critical parameter of the degree of suppression of autoimmunity by these cells (27). To examine regulatory $\mathrm{T}$ cells in the $5 \mathrm{~B} 6$ transgenic mice, we determined the number of $\mathrm{CD} 4^{+} \mathrm{CD} 25^{+} \mathrm{T}$ cells in spleens of $5 \mathrm{~B} 6$ transgenic SJL and B10.S mice by flow cytometry. Our analysis in the 5B6 transgenic mice revealed a generally lower number of $\mathrm{CD}_{25} 5^{+}$cells in the CD $4^{+} \mathrm{T}$ cell population than the expected $10 \%$ in nontransgenic littermates, which is consistent with other TCR transgenic models (7). However, we failed to find significant differences in the number of CD4 ${ }^{+} \mathrm{T}$ cells that coexpressed the CD25 marker between 5B6 transgenic SJL and B10.S mice (data not shown).

We demonstrated that APC subsets from the EAE-resistant B10.S strain had lower basal levels of activation/maturation than

\section{Table 3}

Induction of EAE in 5B6 transgenic B10.S mice via TLR9independent pathways

$\begin{array}{lccc}\text { Administration } & \text { Incidence } & \begin{array}{c}\text { Mean day } \\ \text { of onset }\end{array} & \begin{array}{c}\text { Mean peak } \\ \text { severity }\end{array} \\ \text { LPS } & 1 / 8(13 \%) & 4 & 1.0 \\ \text { PTX } & 8 / 8(100 \%) & 6 \pm 0 & 2.6 \pm 0.3 \\ \text { rm IL-12 } & 1 / 6(17 \%) & 36 & 2.0\end{array}$

5B6 transgenic B10.S mice were injected with LPS $(50 \mu \mathrm{g})$, PTX (75 ng, twice), or rmIL-12 (300 ng, 11 times). Mice were monitored for clinical signs of EAE for 44 days (except for PTX-treated mice). 
did APCs from the autoimmune-susceptible SJL strain. Consistent with this finding, PLP-specific T cells proliferated less when stimulated with PLP139-151 presented by splenic APCs from B10.S mice than with PLP139-151 presented by SJL spleen cells (Figure 3 ). These data indicate that genetic variations in the activation/maturation and function of APCs contribute to EAE susceptibility/resistance. As the 5B6 transgenic SJL and B10.S mice are congenic for the MHC locus, it is likely that the observed difference in $\mathrm{MHC}$ expression is a consequence of differential activation of APCs in the two strains due to differences in non-MHC genes. In this context, it is interesting to note that linkage analysis of susceptibility to EAE in SJL and B10.S mice has revealed susceptibility loci on mouse chromosomes 3 and 8 , which also contain the genes encoding TLR2 and TLR3, respectively $(9,28)$. While TLR2 detects Gram-positive bacteria (29), TLR3 has been implicated in the recognition of double-stranded RNA of viruses $(30,31)$. It remains to be determined whether these receptors of the innate immune system are involved in genetic susceptibility/resistance to EAE. However, we have demonstrated directly that APC subsets from B10.S mice can be activated and expanded by CpG ODN, which is consistent with findings in other systems (17). Cellular responses to $\mathrm{CpG}$ ODNs are signaled through the Toll-like receptor TLR9 (19) and are characterized by proliferation, upregulation of MHC class II and costimulatory molecules, and secretion of proinflammatory cytokines such as TNF- $\alpha$ and IL-12 (32-33). Ichikawa et al. have recently shown that anergy of $\mathrm{T}$ cells could be overcome by stimulating lymph node cells in vitro with anergyinducing antigen in the presence of anti-CD40 or CPG ODN (34). Our data have shown that CPG ODN but not non-CpG ODN

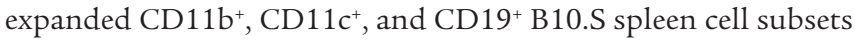
and induced upregulation of MHC class II and CD86 on these APC subpopulations (Figure 4). In vivo administration of CPG but not non-CPG ODN induced EAE in the 5B6 transgenic B10.S mice, demonstrating that peripheral $\mathrm{T}$ cell tolerance can be broken by activation of APCs through the innate immune receptor TLR9 (Table 2). The average day of EAE onset was notably delayed compared with the induction mediated by active immunization protocols using complete Freund's adjuvant and PTX in nontransgenic SJL mice (11). This delay in disease onset may reflect the time that is required in vivo for $\mathrm{CPG} O \mathrm{ODN}$-mediated maturation of APCs and subsequent differentiation of naive transgenic $\mathrm{T}$ cells to effector cells. Nevertheless, activation of APCs was critical for the induction of EAE, as none of the transgenic mice that were injected with non-CPG ODN developed disease.

TLR4-mediated activation of APCs by LPS administration was able to induce a lower incidence of and less-severe EAE in fewer 5B6 transgenic B10.S mice compared with induction with CPG ODN. As both microbial reagents are known to elicit strong IL-12 responses by APCs, we determined whether direct administration of rmIL-12 could induce EAE in 5B6 transgenic B10.S mice. The incidence of EAE by IL-12 injections was lower than that of TLR9mediated EAE induction, but the onset of disease was similar and substantially delayed in both cases. PTX, a microbial agent signaling through as-yet-unidentified receptors, has pleiotropic effects on the immune system, one of which is T cell stimulation via activation of APCs in the absence of IL-12 $(24,25)$. Administration of PTX was most effective in inducing EAE in 5B6 transgenic B10.S mice, with an earlier onset of disease compared with that of CPGinduced EAE. The reasons for the higher incidence and faster onset of EAE by PTX in comparison with CPG administration are not clear but are likely to be the consequence of the diverse biological effects of PTX's acting as an adjuvant in promoting Th differentiation and transendothelial migration of $\mathrm{T}$ cells across the bloodbrain barrier $(35,36)$. As PTX has been reported to stimulate T cells in absence of IL-12 (24), our findings suggest that IL-12 is not essential for the induction of EAE in the $5 \mathrm{~B} 6$ transgenic B10.S mice. This may also explain the relative low incidence of EAE observed in IL-12-treated transgenic mice. IL-23, which is produced predominantly by macrophages and DCs, has recently been reported to be essential for development of EAE in contrast to IL-12 (37). It remains to be determined whether microbial agents such as CPG ODN, LPS, or PTX induce IL-23 production in B10.Sderived APCs and thereby promote development of T cell-mediated autoimmune disease.

In summary, we have found that the low incidence of spontaneous EAE in the $5 \mathrm{~B} 6$ transgenic mice on the B10.S background is not due to a T cell-specific defect but is likely to be controlled by factors that result in activation of APCs. In the presence of activated APCs, self-reactive T cells may differentiate into effector cells and eventually mediate EAE, as we observed in 5B6 transgenic SJL mice. These data suggest that infections resulting in activation of APCs through receptors of the innate immune system such as TLRs or by bacterial toxins such as PTX can tip the balance between peripheral tolerance and autoimmunity in favor of autoimmune disease. This scenario might explain the strong association between infections and relapses in patients with multiple sclerosis (38). These data support the hypothesis that the innate immune system plays a crucial role in maintaining the balance between self tolerance and autoimmunity.

\section{Acknowledgments}

We thank Lea Cefalu, Remy Nazareno, and Nichole Price for technical help and Lindsay Nicholson for helpful comments on the manuscript. This work was supported by grants from the National Institutes of Health (NS 30843, A1 44880, PO1 AI 39671, Javits Award to V.K. Kuchroo), the National Multiple Sclerosis Society (RG 2751 to V.K. Kuchroo; FA 1225A1/1 to H Waldner), and the International Juvenile Diabetes Foundation (10-2000-670 to H. Waldner).

Received for publication July 2, 2003, and accepted in revised form January 29, 2004.

Address correspondence to: Vijay K. Kuchroo, 77 Avenue Louis Pasteur, Center for Neurologic Diseases, Brigham and Women's Hospital and Harvard Medical School, Boston, Massachusetts 02115, USA. Phone: (617) 525-5333; Fax: (617) 525-5566; E-mail: vkuchroo@rics.bwh.harvard.edu.

\footnotetext{
1. Sundvall, M., et al. 1995. Identification of murine loci associated with susceptibility to chronic experimental autoimmune encephalomyelitis. Nat. Genet. 10:313-317.

2. Baker, D., Rosenwasser, O.A., O'Neill, J.K., and Turk, J.L. 1995. Genetic analysis of experimental allergic encephalomyelitis in mice. J. Immunol.
}

155:4046-4051.

3. Wicker, L.S., Todd, J.A., and Peterson, L.B. 1995. Genetic control of autoimmune diabetes in the NOD mouse. Annu. Rev. Immunol. 13:179-200.

4. Encinas, J.A., et al. 1999. QTL influencing autoimmune diabetes and encephalomyelitis map to a $0.15-\mathrm{cM}$ region containing I12. Nat. Genet.
21:158-160.

5. Starr, T.K., Jameson, S.C., and Hogquist, K.A. 2003. Positive and negative selection of T cells. Annu. Rev. Immunol. 21:139-176.

6. Walker, L.S., and Abbas, A.K. 2002. The enemy within: keeping self-reactive $T$ cells at bay in the periphery. Nat. Rev. Immunol. 2:11-19. 
7. Shevach, E.M., McHugh, R.S., Piccirillo, C.A., and Thornton, A.M. 2001. Control of T-cell activation by CD4+ CD25+ suppressor T cells. Immunol. Rev. 182:58-67.

8. Binder, T.A., Clark, R.B., and Goldschneider, I. 1991. Relative susceptibility of SJL/J and B10.S mice to experimental allergic encephalomyelitis is correlated with high and low responsiveness to myelin basic protein. J. Neuroimmunol. 35:31-43.

9. Encinas, J.A., et al. 1996. Genetic analysis of susceptibility to experimental autoimmune encephalomyelitis in a cross between SJL/J and B10.S mice. J. Immunol. 157:2186-2192.

10. Segal, B.M., and Shevach, E.M. 1996. IL-12 unmasks latent autoimmune disease in resistant mice. J. Exp. Med. 184:771-775.

11. Waldner, H., Whitters, M.J., Sobel, R.A., Collins, M., and Kuchroo, V.K. 2000. Fulminant spontaneous autoimmunity of the central nervous system in mice transgenic for the myelin proteolipid proteinspecific T cell receptor. Proc. Natl. Acad. Sci. U. S. A. 97:3412-3417.

12. Chu, R.S., Targoni, O.S., Krieg, A.M., Lehmann, P.V., and Harding, C.V. 1997. CPG oligodeoxynucleotides act as adjuvants that switch on $\mathrm{T}$ helper 1 (Th1) immunity. J. Exp. Med. 186:1623-1631.

13. Lutz, M.B., et al. 1999. An advanced culture method for generating large quantities of highly pure dendritic cells from mouse bone marrow. J. Immunol. Methods. 223:77-92.

14. Kuchroo, V.K., et al. 1992. Experimental allergic encephalomyelitis mediated by cloned $\mathrm{T}$ cells specific for a synthetic peptide of myelin proteolipid protein. Fine specificity and $\mathrm{T}$ cell receptor $\mathrm{V}$ beta usage. J. Immunol. 148:3776-3782.

15. Berg, L.J., et al. 1989. Antigen/MHC-specific T cells are preferentially exported from the thymus in the presence of their MHC ligand. Cell. 58:1035-1046.

16. Mellman, I., and Steinman, R.M. 2001. Dendritic cells: specialized and regulated antigen processing machines. Cell. 106:255-258.

17. Hacker, H., et al. 1998. CpG-DNA-specific activation of antigen-presenting cells requires stress kinase activity and is preceded by non-specific endocytosis and endosomal maturation. EMBO J. 17:6230-6240.

18. Sparwasser, T., et al. 1998. Bacterial DNA and immunostimulatory $\mathrm{CPG}$ oligonucleotides trigger maturation and activation of murine dendritic cells. Eur. J. Immunol. 28:2045-2054.

19. Hemmi, H., et al. 2000. A Toll-like receptor recognizes bacterial DNA. Nature. 408:740-745.

20. Pape, K.A., Khoruts, A., Mondino, A., and Jenkins, M.K. 1997. Inflammatory cytokines enhance the in vivo clonal expansion and differentiation of antigen-activated CD4+ T cells. J. Immunol. 159:591-598.

21. Hoshino, K., et al. 1999. Cutting edge: Toll-like receptor 4 (TLR4)-deficient mice are hyporesponsive to lipopolysaccharide: evidence for TLR4 as the Lps gene product. J. Immunol. 162:3749-3752.

22. Linthicum, D.S., Munoz, J.J., and Blaskett, A. 1982. Acute experimental autoimmune encephalomyelitis in mice. I. Adjuvant action of Bordetella pertussis is due to vasoactive amine sensitization and increased vascular permeability of the central nervous system. Cell. Immunol. 73:299-310.

23. Spangrude, G.J., Braaten, B.A., and Daynes, R.A. 1984. Molecular mechanisms of lymphocyte extravasation. I. Studies of two selective inhibitors of lymphocyte recirculation. J. Immunol. 132:354-362.

24. Wakatsuki, A., Borrow, P., Rigley, K., and Beverley, P.C. 2003. Cell-surface bound pertussis toxin induces polyclonal $\mathrm{T}$ cell responses with high levels of interferon-gamma in the absence of interleukin12. Eur. J. Immunol. 33:1859-1868.

25. Shive, C.L., Hofstetter, H., Arredondo, L., Shaw, C., and Forsthuber, T.G. 2000. The enhanced antigenspecific production of cytokines induced by pertussis toxin is due to clonal expansion of T cells and not to altered effector functions of long-term memory cells. Eur. J. Immunol. 30:2422-2431.

26. Trinchieri, G. 1995. Interleukin-12: a proinflammatory cytokine with immunoregulatory functions that bridge innate resistance and antigen-specific adaptive immunity. Annu. Rev. Immunol. 13:251-276.

27. Sakaguchi, S., Sakaguchi, N., Asano, M., Itoh, M., and Toda, M. 1995. Immunologic self-tolerance maintained by activated T cells expressing IL-2 receptor alpha-chains (CD25). Breakdown of a single mechanism of self-tolerance causes various autoimmune diseases. J. Immunol. 155:1151-1164.

28. Butterfield, R.J., et al. 1998. New genetic loci that control susceptibility and symptoms of experimental allergic encephalomyelitis in inbred mice. J. Immunol. 161:1860-1867.

29. Alexopoulou, L., Holt, A.C., Medzhitov, R., and Flavell, R.A. 2001. Recognition of double-stranded RNA and activation of NF-kappaB by Toll-like receptor 3. Nature. 413:732-738.

30. Werts, C., et al. 2001. Leptospiral lipopolysaccharide activates cells through a TLR2-dependent mechanism. Nat. Immunol. 2:346-352.

31. Hirschfeld, M., et al. 2001. Signaling by toll-like receptor 2 and 4 agonists results in differential gene expression in murine macrophages. Infect. Immun. 69:1477-1482.

32. Stacey, K.J., Sweet, M.J., and Hume, D.A. 1996. Macrophages ingest and are activated by bacterial DNA. J. Immunol. 157:2116-2122.

33. Sparwasser, T., et al. 1997. Bacterial DNA causes septic shock. Nature. 386:336-337.

34. Ichikawa, H.T., Williams, L.P., and Segal, B.M. 2002. Activation of APCs through CD40 or Toll-like receptor 9 overcomes tolerance and precipitates autoimmune disease. J. Immunol. 169:2781-2787.

35. Hou, W., et al. 2003. Pertussis toxin enhances Th1 responses by stimulation of dendritic cells. J. Immunol. 170:1728-1736.

36. Adamson, P., et al. 2002. Lymphocyte trafficking through the blood-brain barrier is dependent on endothelial cell heterotrimeric G-protein signaling. FASEB J. 16:1185-1194.

37. Cua, D.J., et al. 2003. Interleukin-23 rather than interleukin-12 is the critical cytokine for autoimmune inflammation of the brain. Nature. 421:744-748.

38. Buljevac, D., et al. 2002. Prospective study on the relationship between infections and multiple sclerosis exacerbations. Brain 125:952-960. 